With a non-uniform material $\sum \mu_{k} d n_{k}$ is zero along a streamline if there is no diffusion and if any chemical reaction is reversible. Thus, since diffusion and irreversibility of chemical reaction are real-fluid effects associated with varying composition, no new feature appears in the generalized form of Crocco's vorticity law.

The vorticity law is most useful if $h_{0}$ and the $n_{k}$ 's are constant over the entire flow field; in this case the entropy gradient may be obtained directly from the vorticity or the lateral components of the vorticity from the entropy gradient. However, with no real-fluid effects present and with sufficient knowledge of the flow field the vorticity law still gives a useful relation between vorticity and entropy gradient, as then $h_{0}$ is a function only of the streamline and the $n_{k}$ 's may in principle be obtained, and the lateral gradients of these quantities may be calculated.

In a binary mixture we may set

$$
\begin{gathered}
c=n_{1} M_{1}, \\
1-c=n_{2} M_{2}, \\
\mu=\frac{\mu_{1}}{M_{1}}-\frac{\mu_{2}}{M_{2}},
\end{gathered}
$$

where $M_{1}$ and $M_{2}$ are the molecular weights (molar mass) of the two components. With this notation we have

$$
\sum \mu_{k} d n_{k}=\mu d c
$$

and Crocco's vorticity law takes the form

$$
\mathbf{q} \times(\nabla \times \mathbf{q})=\nabla h_{0}-T \nabla s-\mu \nabla c .
$$

\title{
REFERENCE
}

[1] L: Crocco, Ein neue Stromfunktion für die Erforschung der Bewegung der Gase mit Rotation, ZAMM 17, 1-7 (1937)

\section{THE SCHWARZIAN DERIVATIVE AND THE APPROXIMATION METHOD OF BRILLOUIN*}

BY AUREL WINTNER (The Johns Hopkins University)

1. Let the coefficient function of the differential equation

$$
x^{\prime \prime}+f^{2}(t) x=0
$$

be the square of a positive function $f(t)$ which is given, and possesses a continuous second derivative, for large positive $t$, say for $t_{0} \leqq t<\infty$. Then the function

$$
\varphi(t)=\int_{t_{0}}^{t} f(s) d s
$$

is strictly increasing and has a continuous third derivative, whereas the function

\footnotetext{
${ }^{*}$ Received January 17, 1957.
} 


$$
4 F(t)=3 f^{\prime 2}(t) / f^{4}(t)-2 f^{\prime \prime}(t) / f^{3}(t)
$$

exists and is continuous.

In view of (2), the definition of $F(t)$ is equivalent to

$$
F(t)=-2 f^{2}(t)\{\varphi(t)\},
$$

if \{\} is the symbol of the differential parameter of Lagrange-Schwarz,

$$
\{\varphi\}=\left(\varphi^{\prime \prime} / \varphi^{\prime}\right)^{\prime}-\left(\varphi^{\prime \prime} / \varphi^{\prime}\right)^{2} / 2 .
$$

It is also seen from (3) that

$$
F(t) f(t)=k(t) k^{\prime \prime}(t), \text { where } k(t)=[f(t)]^{-1 / 2} .
$$

2. If $t \rightarrow \infty$, then the function (2), being increasing, tends either to a finite limit $\varphi(\infty)$ or to $\varphi(\infty)=\infty$. Suppose that the latter is the case,

$$
\int^{\infty} f(t) d t=\infty \quad(f>0),
$$

and that the function (6) is absolutely integrable,

$$
\int^{\infty}|F(t)| f(t) d t<\infty \quad(f>0) .
$$

The following theorem was proved ten years ago ${ }^{1}$ (and, in certain particular cases of it, was repeatedly rediscovered ${ }^{2}$ in the meantime):

For large positive $t$, let $f(t)$ be a positive, twice continuously differentiable function satisfying conditions (7) and (8), where $F(t)$ is defined by (3). Then the general solution of the differential equation (1) is the superposition of two linearly independent solutions which are asymptotic (as $t \rightarrow \infty$ ) to the real and imaginary parts of the function

$$
y(t)=[f(t)]^{-1 / 2} \exp \left[i \int_{t_{0}}^{t} f(s) d s\right] .
$$

In addition, differentiation of this asymptotic representation is allowed.

In other words, the assumptions (7) and (8) are sufficient in order that the general solution of the differential equation (1) be of the form $x(t)=c_{1} x_{1}(t)+c_{2} x_{2}(t)$, where, as $t \rightarrow \infty$,

$$
x_{1}(t) \sim y_{1}(t), \quad x_{2}(t) \sim y_{2}(t) \quad \text { and } \quad x_{1}^{\prime}(t) \sim y_{1}^{\prime}(t), \quad x_{2}^{\prime}(t) \sim y_{2}^{\prime}(t)
$$

if the real functions $y_{1}(t), y_{2}(t)$ are defined by placing

$$
y_{1}(t)+i y_{2}(t)=[f(t)]^{-1 / 2} \exp [i \varphi(t)] .
$$

3. The point in the theorem just quoted was the generality of its assumptions (and, correspondingly, the comparative simplicity of its proof), since, under assumptions

${ }^{1}$ A. Wintner, Phys. Rev. 72, 516-517 (1947).

2See, e.g., M. Zlámal, Czechoslov. Math. J. 6 (81), 75-93 (1956) (in German, with a Russian abstract), where one of the books of R. Bellman (1953) is mentioned. The main result of Zlámal's Chap. II (Theorem 6 on p. 83, along with the proof on pp. 84-85) is the simplest of the corollaries of the note in the Phys. Rev., referred to in the preceding footnote; see the general theorem italicized above, which shows that the problem is independent of Zlámal's convexity assumption. 
more stringent than the pair of conditions (7) and (8), the asymptotic form (9) was well known from the mathematical as well as from the physical literature of the subject. ${ }^{8}$ The proof of the theorem depended on a combination of two steps: $(\alpha)$ the standard use of the formal substitution of the Liouville and Riemann, ${ }^{8}$ and $(\beta)$ the application of an "Abelian lemma," which runs as follows."

If $\epsilon=\epsilon(\varphi)$ is defined, for large positive $\varphi$, as an arbitrary continuous (possibly not even real-valued) function which is absolutely integrable,

$$
\int^{\infty}|\epsilon(\varphi)| d \varphi<\infty,
$$

then the general solution $z=z(\varphi)$ of the differential equation

$$
d^{2} z / d \varphi^{2}+[1+\epsilon(\varphi)] z=0
$$

(as well as the derivative, $d z(\varphi) / d \varphi$, of every solution) has, as $\varphi \rightarrow \infty$, the same asymptotic behavior as what results in the trivial case, $\epsilon(\varphi) \equiv 0$, of (13); so that

$$
z(\varphi)-\left(c_{1} \sin \varphi+c_{2} \cos \varphi\right) \rightarrow 0, \quad d z(\varphi) / d \varphi-\left(c_{1} \cos \varphi-c_{2} \sin \varphi\right) \rightarrow 0
$$

as $\varphi \rightarrow \infty$. In the application of this Abelian lemma, the independent variable $\varphi$ must be chosen to be the function defined by (2) or (4) above. The rôle of step ( $\alpha$ ) was precisely the reduction of (1) to (13), where $\epsilon(\varphi)$ is "small" (for large $\varphi$ ) in an appropriate sense, whereas step $(\beta)$ consisted in assuring that condition (12) is a sufficient "degree of smallness" for the asymptotic form (14) of the general solution of (13).

In what follows, a more general and less artificial approach will be taken to the asymptotic behavior of the solutions $x(t)$ of $(1)$ and of their derivatives. The improvements will depend on a formal fact pointed out by Brillouin. ${ }^{7}$

\$For references, see G. Sansone, Equazioni differenziali nel campo reale vol. 2, Bologna, 1949, p. 53; see also pp. 101-110 of Titchmarsh's book, referred to in footnotes.

4See G. Sansone, op. cit., vol. 2, pp. 68-70.

'B. Riemann, Gesammalte mathematische Werke, 2nd ed., Leipzig, 1892, pp. 335-336, or the presentation in E. G. C. Poole's Theory of linear differential equations, Oxford, 1936, pp. 121-122; see also E. C. Titchmarsh, Eigenfunction expansions associated with second-order differential equations, Oxford, 1946, p. 102.

${ }^{6}$ A proof, based on successiive approximations, was given by H. Weyl, Gruppentheorie und Quantenmechanik, Leipzig, 1928, pp. 61-63. Actually, as pointed out in my note in the Phys. Rev., referred to above $^{1}$, the same proof is contained already in an important paper of M. Bôcher (1900). For another arrangement of the proof, a proof based on the "Abelian" aspect, see A. Wintner, Amer. J. Math. 69, 251-272 (1948).

As shown recently [A. Wintner, Amer. J. Math. 78, 895-897 (1956)], the latter proof is such as to lead to simple criteria which are substantially more general than (12) and are, nevertheless, sufficient in order that (14) should hold for the general solution of (13).

As pointed out at the end of the paper just mentioned, the question of asymptotic behavior is stricter than that of stability (boundedness). For an application of the Loiuville-Riemann transformation to the latter question, see U. Barbuti, Annali di Pisa, ser. 3, 8, 81-91 (1954), where, with regard to the question of stability, recent papers of G. Ascoli (1947), L. A. Gusarov (1949) and U. Barbuti (1952) are cited.

${ }^{7}$ L. Brillouin, Quart. Appl. Math. 6, 166-178 (1948) and 7, 363-380 (1950) (in particular p. 172 and p. 364 respectively); see also L. Brillouin and M. Parodi, Propagation des ondes dans les milieux périodiques, Paris, 1956, p. 216. Strictly speaking, in what Brillouin is interested is, not the asymptotic behavior, for indefinitely large $t$, of the solutions $x(t)$ of a fixed differential equation (1), but a situation in which the $t$-range is limited but the differential equation (1), instead of being fixed, contains a variable parameter. 
4. For large positive $t$, let $f(t)$ be any, perhaps not even real-valued, twice continuously differentiable function which is nowhere zero,

$$
f(t) \neq 0 \text { for } t_{0} \leqq t<\infty
$$

[which, if $f(t)$ is real-valued or purely imaginary, means that

$$
f^{2}(t)>0 \text { or } f^{2}(t)<0
$$

throughout]. Then, with either of the continuous determinations of the square root occurring in it, Eq. (9) defines a twice continuously differentiable function

$$
y(t) \neq 0 \text { for } t_{0} \leqq t<\infty .
$$

Two differentiations of the definition (9) show that

$$
y^{\prime \prime}(t)+\left[f^{2}(t)-f^{2}(t) F(t)\right] y(t)=0
$$

is an identity, if $F(t)$ is defined by (3) [that is, by (4) and (2) together or, equivalently, by (6)]. Starting with the identity (18), Brillouin observes that the function (9) can be expected to furnish an approximation to a solution $x(t)$ of (1) when the coefficient, [ ], of $y(t)$ in (18) is "sufficiently close" to the coefficient, $f^{2}(t)$, of $x(t)$ in (1), that is, if $f^{2}(t) F(t)$ is "sufficiently small.",

The issue is, of course, the specification of conditions under which such a "degree of smallness" (for large $t$ ) will actually be realized. But it turns out that such a specification can be obtained, in explicit terms, from a general criterion. The criterion in question is as follows. ${ }^{\circ}$

If $y=y_{1}(t)$ and $y=y_{2}(t)$ are two, linearly independent, solutions of a differential equation

$$
y^{\prime \prime}+g(t) y=0
$$

in which $g(t)$, where $t_{0} \leqq t<\infty$, is any continuous function, and if $h(t)$, where $t_{0} \leqq t<\infty$, is any continuous function having the property that the corresponding differential equation

$$
x^{\prime \prime}+h(t) x=0
$$

is so "close" (as $t \rightarrow \infty$ ) to the differential equation (19) that

$$
\int^{\infty}\left(\left|y_{1}(t)\right|^{2}+\left|y_{2}(t)\right|^{2}\right)|g(t)-h(t)| d t<\infty
$$

holds, then the two differential equations are asymptotically equivalent, in the following sense. The differential equation (20) will possess two linearly independent solutions, $x=x_{1}(t)$ and $x=x_{2}(t)$, which, along with their derivatives, will satisfy the asymptotic relations (10) as $t \rightarrow \infty$.

5. In order to combine this criterion with Brillouin's method, let (18) and (1) be identified with (19) and (20) respectively (so that $|g-h|$ becomes $\left.\left|f^{2} F\right|\right)$ and, under the assumption that $f(t)$ is positive, let $y_{1}(t)$ and $y_{2}(t)$ be those (real-valued) solutions

IIn this regard, see the comments made at the end of the preceding footnote.

'A. Wintner, Quart. Appl. Math. 15, 428-430 (1957). 
of (18) for which $y_{1}(t)+i y_{2}(t)$ becomes the (complex-valued) function (9). Then the condition (21) reduces to the convergence of the integral

$$
\int^{\infty}|y(t)|^{2} f^{2}(t)|F(t)| d t,
$$

since $\left|y_{1}\right|^{2}+\left|y_{2}\right|^{2}=|y|^{2}$ and $|g-h|=|F| f^{2}$. But the convergence of this integral is equivalent to the condition (8), since $|y|^{2} f^{2}=f$, by the definition, (9), of $y$, where $|\exp |=1$.

Accordingly, the general criterion of Sec. 4 supplies, as a corollary, not only the theorem quoted in Sec. 2, but also an improvement of that theorem. In fact, it is now seen that the assumption (7) of the theorem italicized above can be omitted, that is, only the other assumption, (8), of the theorem is needed.

6. This reduction of the assumptions of the theorem could have been proved directly. The main point was, however, the possibility of a straightforward approach, one replacing the formalism of the Liouville-Riemann substitution by its simplified version, the formal identity of Brillouin.

The spurious character of the restriction (7) is indicated even by Brillouin's elemen tary case. In fact, the latter results by assigning the following condition for the coefficient function of (1):

$$
F(t)=0 \text { for } t_{0}<t<\infty .
$$

In view of the definition (3), the assignment (22) represents a differential equation of second order for $f(t)$, and Brillouin's approximation theory (loc. cit.) depends on the circumstance that the general solution of the (non-linear) differential equation (22) is

$$
f(t)=C_{1} /\left(t+C_{2}\right)^{2},
$$

$\left(C_{1}\right.$ and $C_{2}$ are the integration constants), as is readily verified by direct substitution. Clearly, (22) means that the differential equation (18) is not only "close" to, but is identical with, the "perturbed" differential equation (1) and, correspondingly, condition (8) is certainly satisfied. But condition (7) happens to be violated. In fact, if (without loss of generality) $C_{1}=1$ and $C_{2}=0$, then the general solution, (23), of (22) reduces to $f(t)=t^{-2}$, and so

$$
\int_{t_{0}}^{\infty} f(t) d t=\int_{t_{0}}^{\infty} t^{-2} d t<\infty \quad\left(t_{0}>0\right)
$$

holds, instead of (7). The exact solution of this case of (1) is supplied by the real and imaginary parts of the function

$$
x(t)=t \exp (i / t), \quad\left(0<t_{0} \leqq t<\infty\right),
$$

since (9) reduces to this function for the choice $f(t)=t^{2}$ (if $i$ is replaced by $-i$ and a constant factor, introduced by the arbitrary integration limit $t_{0}>0$, is disregarded). 